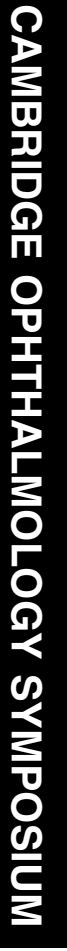

${ }^{1}$ Department of Pharmaceutical Sciences, College of Pharmacy, University of Nebraska Medical Center, Omaha, NE, USA

${ }^{2}$ Department of Ophthalmology, College of Medicine, University of Nebraska Medical Center, Omaha, NE, USA

${ }^{3}$ Department of Veterinary Clinical Sciences,

Comparative Ophthalmology, College of Veterinary Medicine, The Ohio State University, Columbus, OH, USA

Correspondence: PF Kador, College of Pharmacy, University of Nebraska Medical Center, 986025 Nebraska Medical Center, Omaha, NE 68198-6025, USA Tel: + 1402559 9261;

Fax: + 14025599543 .

E-mail: pkador@unmc.edu

Received: 18 January 2008

Accepted in revised form:

18 January 2008

Published online:

14 March 2008

\section{Asteroid hyalosis: pathogenesis and prospects for prevention}

\begin{abstract}
Asteroid hyalosis (AH) is a common degenerative process in which fatty calcium globules collect within the vitreous humour. The condition rarely causes visual disturbances, and surgical removal is only rarely required. The presence of $\mathrm{AH}$ has been associated with systemic diseases such as diabetes; however, research in this area has been hampered by the lack of an animal model of AH. Recently, we have reported that $\mathrm{AH}$ occurs in galactose-fed beagles that develop the advanced stages of diabetes-like retinopathy. Comparisons of vitreous humour containing asteroid bodies (ABs) collected from these galactose-fed beagles and vitreous samples from age-matched normal beagles without ABs indicate that ABs contain calcium and phosphorous. Subtraction analysis of chloroform extracts of the vitreous samples by electrospray mass spectroscopy resulted in the identification of the quasimolecular ion of 1,2dipalmitoyl-glycero-3-phosphoethanolamine (DPPE) as the main component of ABs. Since several reports indicate that $\mathrm{ABs}$ are composed of lipid-calcium complexes, we have proposed that the main component of $\mathrm{ABs}$ in the galactose-fed dogs with $\mathrm{AH}$ is a quasimolecular ion of DPPE in which two molecules of DPPE are complexed through their phosphates groups with calcium. We suggest that these lipid components diffuse into the vitreous from the degenerating retinas of these dogs.

Eye (2008) 22, 1278-1285; doi:10.1038/eye.2008.35; published online 14 March 2008
\end{abstract}

Keywords: vitreous; diabetes; asteroid hyalosis; asteroid bodies; animal models; galactosemic dog

\section{Introduction}

The vitreous is a highly hydrated gel-like substance that provides structural support to
PF Kador ${ }^{1,2}$ and M Wyman ${ }^{3}$

the eyeball while providing a clear

unobstructed path for light to reach the retina. In addition, it hinders the forward diffusion of oxygen from the retinal blood supply to the anterior segment of the eye where it can cause oxidation damage to the lens. The vitreous is an extracellular matrix that is composed of collagen fibrils with the carbohydrate polymer hyaluronan (hyaluronic acid) serving as the major component between the fibrils. ${ }^{1}$ With age, it may partially liquefy as a result of hyaluronan breakdown associated with oxidative stress., ${ }^{2,3}$ Once liquefied or surgically removed, the vitreous does not reform significantly.

Opacities may form in the clear vitreous as a result of developmental abnormalities, injury, or disease. Developmental opacities present from birth are the remnants of the hyaloid system that are located in Cloquet's canal, whereas degenerative opacities, associated with inflammatory debris, often appear after ocular inflammation, ocular haemorrhage, posterior vitreous detachment, or vitreous haemorrhage. Ocular trauma or inflammation may also result in the accumulation of glittering cholesterol crystals in the vitreous humour along with the anterior chamber and subretinal space (synchisis scintillans). However, the most common clinically observed degenerative opacity of the vitreous is asteroid hyalosis (AH). Accurately differentiated from synchisis scintillans in the late 1800s, it was first called asteroid hyalitis; however, the name was changed in the 1960s to AH because of the absence of inflammatory changes. $\mathrm{AH}$ is characterized by spherical white bodies that move along with the vitreous humour when the eye moves. They are present unilaterally in approximately $75 \%$ of patients and are more predominant in men than women unilaterally. ${ }^{4-6}$ Although their presence is obvious upon ocular examination and in severe cases make retinal examination difficult, patients are usually unaware of $\mathrm{AH}$ because the asteroid bodies 
(ABs) rarely cause visual disturbances. Surgical removal of these opacities is usually not required unless $\mathrm{AH}$ compromises the patient's vision.

\section{Pathogenesis of AH}

Although $\mathrm{AH}$ has been associated with several systemic diseases, the cause(s) and mechanism(s) of AH formation remain unknown. Three major studies conclude that $\mathrm{AH}$ is primarily associated with ageing. In the Beaver Dam Study of Wisconsin where 4952 patients were examined, $\mathrm{AH}$ was present in $1.2 \%$ of the population with prevalence increasing from $0.2 \%$ in subjects $43-54$ years of age to $2.9 \%$ in those aged $75-86$ years. ${ }^{6} \mathrm{AH}$ was primarily unilateral and was more prevalent in men. The Australian Blue Mountains Eye Study of 3654 patients aged 49-97 years confirmed a similar 1\% prevalence of $\mathrm{AH} .{ }^{5}$ In this group, the prevalence of $\mathrm{AH}$ also increased with age from $0 \%$ of persons $49-55$ years of age to $2.1 \%$ of persons aged 75-97 years, and $\mathrm{AH}$ was again more prevalent in men. A higher, $1.96 \%$, prevalence of $\mathrm{AH}$ was observed in the third study from the University of California at Los Angeles (UCLA) autopsy eye database from 1965 to 2000 where 10801 patients were examined. ${ }^{4}$ This retrospective study, which demographically matched the characteristics of the US population on the 2000 census, also showed an inverse relationship between $\mathrm{AH}$ and posterior vitreous detachment.

Although these three major studies found $\mathrm{AH}$ to primarily correlate with ageing, others suggest that $\mathrm{AH}$ is associated with diabetes mellitus (DM), ${ }^{7-16}$

hypertension, ${ }^{14,17}$ hypercholesteraemia or lipidemia, ${ }^{7,15,16}$ or increased serum calcium levels. ${ }^{18,19}$ Of these, a link to $\mathrm{DM}$ is most often cited; however, this link is also the most controversial. Although AH predominantly occurs unilaterally, ${ }^{4-6} \mathrm{AH}$ should be bilateral if linked to systemic diseases such as DM because each eye is equally exposed to hyperglycaemia. Bilateral 'trends' of AH have been suggested among diabetic populations ${ }^{11}$ and the UCLA autopsy study, ${ }^{4}$ which observed a statistical association between diabetes and bilateral $\mathrm{AH}$ before adjusting the data for age and sex. However, in the UCLA study, AH was present only in 20 of 780 diabetics. $\mathrm{AH}$ formation in diabetics has been suggested to be secondary to retinal vascular changes. ${ }^{10}$ The overall relationship between $\mathrm{DM}$ and $\mathrm{AH}$ is further complicated by data suggesting that $\mathrm{AH}$ may arrest the process of vitreous collapse or contraction in eyes with proliferative diabetic retinopathy. 8,20

\section{Structure and composition of ABs}

The structure and composition of ABs is currently unknown. Studies to date have utilized clinically obtained human AH. However, the small size and amounts of ABs available for study, along with their strong adherence to vitreous humour, have limited applications to histological and microscopic techniques. Histological studies indicate that unlike the vitreous humour, ABs readily stain for the presence of lipids (Sudan black B and oil red 0) and calcium (von Kossa). However, ABs do not dissolve in common fat solvents. This led Verhoeff ${ }^{21}$ in the early 1920s to postulate that ABs are calcium soaps with calcium primarily bound to the fatty acid carboxylate groups. Forty years later, Rodman et $a^{22}$ confirmed that calcium is bound to the main acidic lipid components of ABs. However, its behaviour is not consistent with fatty acid calcium salts because the ABs did not dissolve in glacial acetic acid or $0.8 \% \mathrm{HCl}$ but did dissolve in methanolic $\mathrm{HCl}$. Moreover, two types of $\mathrm{AH}$ were observed. In the first, Type I AH, the ABs were suspended in and surrounded by vitreous fibrils. The ABs appeared composed of two zones, a large inner zone filled with birefringent particles and a thin cortical rim composed of condensed nonbirefringent vitreous particles. In the second, Type II AH, the ABs were surrounded by cellular elements and this whole complex was enmeshed with vitreous fibrils or inflammatory tissue. The appearance of these two types of ABs suggest potential differences in their pathogenic mechanism(s); however, both types have been documented to be present in the same patient. ${ }^{12}$ Moreover, despite the apparent link with inflammatory tissues, uveitis is not associated with $\mathrm{AH}$.

In the 1970s, analyses of ABs by X-ray microanalyser revealed that phosphate, in addition to calcium, is present as a major element. ${ }^{23}$ This suggests that calcium is present in $\mathrm{ABs}$ in the form of a calcium phosphate complex (apatite) rather than as fatty acid calcium salts. Electron microscopy revealed the presence of 'satellite' ABs composed of a number of round particles of equal size and in symmetrical arrangement. These particles appeared embedded in a matrix, which merged with the surrounding vitreous. These observations led March and $\mathrm{Shoch}^{23}$ to minimize the lipid character of ABs and to suggest that ABs may simply be a physicochemical phenomenon or shift of a part of the normal vitreous from the liquid to a solid phase. In this process, 'satellite' asteroids are initially crystallized from 'seeds' formed in the supersaturated vitreous. These 'satellites' then conglomerate in the solid phase to form the larger, experimentally observed ABs. The basic processes leading to this hypothetical supersaturated solution were not identified.

In contrast to the suggestion that $\mathrm{ABs}$ are derived from the vitreous, which is naturally low in lipids, Streeten ${ }^{24}$ reported in 1982 that ABs must be derived from sources exogenous to the vitreous. This was based on 
observations that large quantities of complex lipids and calcium are present in ABs. He reported that larger ABs lying in a homogeneous background matrix are composed of intertwined ribbons of multilaminar membranes with a 6-nm periodicity characteristic of complex lipids, especially phospholipids. Interspersed throughout the ABs are clumps of dense material with swirls of thin membranous strands often surrounding them. The structures of the smaller ABs suggest that the lipid ribbons result from the deposition of successive lipid layers on thin membranes so that various degrees of lipid were contained. In ABs of all sizes, energydispersive $\mathrm{X}$-ray analysis indicated that calcium and phosphorus were the main elements, and electrondiffraction structural analysis indicated that calcium hydroxyapatite and possibly other forms of calcium phosphate crystals are present in the focal densities of the ABs. Others using electron microscopy have subsequently reported that $A B s$ contain interwined ribbons of multilaminar membranes with a periodicity (10-60 $\mathrm{A})$ that is characteristic of complex lipids, especially phospholipids, along with calcium and phosphate. ${ }^{12,25}$ Miller $e t a^{26}$ suggested that ABs are not true crystals but rather liquid crystals of phospholipids in the vitreous humour. This was based on their ability under appropriate conditions of fixation and highresolution transmission electron microscopy to observe that ABs had a lamellar arrangement with a periodicity of $4.6 \mathrm{~nm}$, which is typical of liquid crystalline phases of lipids in water.

After another nearly 20-year lapse, two recent publications provide additional evidence for the apatitelike nature of $\mathrm{ABs}^{27,28}$ Using element mapping with electron spectroscopic imaging, Winkler and Lunsdorf ${ }^{27}$ in 2001 demonstrated that calcium, phosphorus, and oxygen are homogeneously distributed in $\mathrm{ABs}$ and that the electron energy loss spectra of these elements shows details similar to those found for hydroxyapatite. Moreover, they found that $\mathrm{ABs}$ were time dependent and degraded in the presence of the calcium-specific chelator Na-EGTA. Winkler and Lunsdorf ${ }^{27}$ also observed by immunofluorescence that chondroitin-6-sulphate is present at the periphery of $\mathrm{ABs}$ and that part of the inner matrix of $\mathrm{ABs}$ is composed of carbohydrates specific for hyaluronic acid.

Komatsu $e t a l^{28}$ in 2003 confirmed the apatite-like structure of $\mathrm{ABs}$ by comparing the surface patterns of artificial hydroxyapatite produced in vitro with simulated body fluid containing $\mathrm{Na}^{+}, \mathrm{K}^{+}, \mathrm{Ca}^{+2}, \mathrm{Mg}^{+2}, \mathrm{HCO}_{3}^{-}$, $\mathrm{Cl}^{-}, \mathrm{HPO}_{4}^{-2}$, and $\mathrm{SO}_{4}^{-2}$ to those of $\mathrm{AH}$ particles on collagen-coated styrene plates. They also reported that the ABs by scanning electron microscopy appeared as snowballs with a pumice stone-like structure whose surfaces were covered with a clotted matrix of vitreous substances. From these observations, they suggested that $\mathrm{AH}$ is produced not only by changes of ionic tension in the vitreous fluid but also by changes of vitreous matrix associated with the ageing process or disease. Since the observed structural characters of the ABs resemble stones in the living body (lithiasis), Komatsu et al ${ }^{28}$ suggested that $\mathrm{AH}$ is a condition of lithiasis in the body.

Although studies have focused on the structure and elemental composition of ABs, little progress has been made on identifying the actual biochemical components present in ABs. Such analyses are hampered by contamination since $\mathrm{ABs}$ are virtually impossible to separate from the surrounding vitreous. Using the techniques of thin layer and gas chromatography available in the 1960s, Feldman ${ }^{29}$ reported that the lipid composition of $\mathrm{ABs}$ from humans contains $21 \%$ cholesterol, $5 \%$ cholesteryl esters, $38 \%$ sphingomyelin, $23 \%$ ceramide dihexoside, $10 \%$ cerebroside, and trace amounts of triglycerides and lecithin (3\% of lipids were unidentified). Until now, no other investigators have addressed this problem.

Currently, Lin $e t a l^{30}$ have reported the presence of $\beta$-carotene in $\mathrm{ABs}$ in one of two patients with $\mathrm{AH}$ whose $A B s$ were obtained by vitrectomy and examined by confocal Raman microspectroscopy and high pressure liquid chromatography (HPLC) analysis. $\beta$-Carotene was identified by Raman spectroscopy in the presence of two unique peaks at 1525 and $1158 \mathrm{~cm}^{-1}$. Subsequently, the presence of $\beta$-carotene was confirmed by comparison of HPLC retention times with standard $\beta$-carotene.

\section{The galactose-fed dog}

In addition to humans, $\mathrm{AH}$ has been observed in dogs, ${ }^{31,32}$ chinchillas, ${ }^{33}$ cats $^{32}$ and rabbits. ${ }^{34}$ While none of these specific animals consistently develop bilateral $\mathrm{AH}$, we have observed that young (9-month old or less) male beagles fed a diet containing 30\% galactose for 42 months all developed AH. ${ }^{35}$ Galactose feeding activates the enzyme aldose reductase, which is the first step of the Sorbitol Pathway in which excess glucose is converted to fructose through the intermediate sugar alcohol sorbitol. Under hyperglycaemic conditions, the activation of aldose reductase results in the intracellular accumulation of sorbitol and this accumulation has been linked to the development of diabetic complications, which in the eye include cataract, retinopathy, and keratopathy. In galactose-fed animals, intracellular galactose is more rapidly converted to galactitol by aldose reductase than glucose is to sorbitol. Unlike sorbitol, galactitol is not further metabolized. The net result is a more rapid and higher intracellular accumulation of sugar alcohol in the galactose-fed compared to diabetic animal, and this results in a more rapid and more severe development of 
Table 1 Development of diabetes-like ocular lesions in 9-month old beagles fed $30 \%$ galactose diet

\begin{tabular}{ll}
\hline $\begin{array}{l}\text { Months } \\
\text { diet }\end{array}$ & Ocular lesions \\
\hline $2-6$ & Cataract \\
$19-24$ & Pericyte ghost formation \\
$27-31$ & Microaneurysms \\
$33-36$ & Dot and blot haemorrhages \\
$36-48$ & Confluent haemorrhages, areas of nonperfusion \\
42 & Asteroid hyalosis \\
56 & IRMA \\
60 & Occluded arterioles; A-V shunts; exudates; gliosis of \\
66 & nerve fibre layer \\
72 & Intravitreal haemorrhage; partial posterior vitreous \\
& Proliferative retinopathy, NVD \\
\hline
\end{tabular}

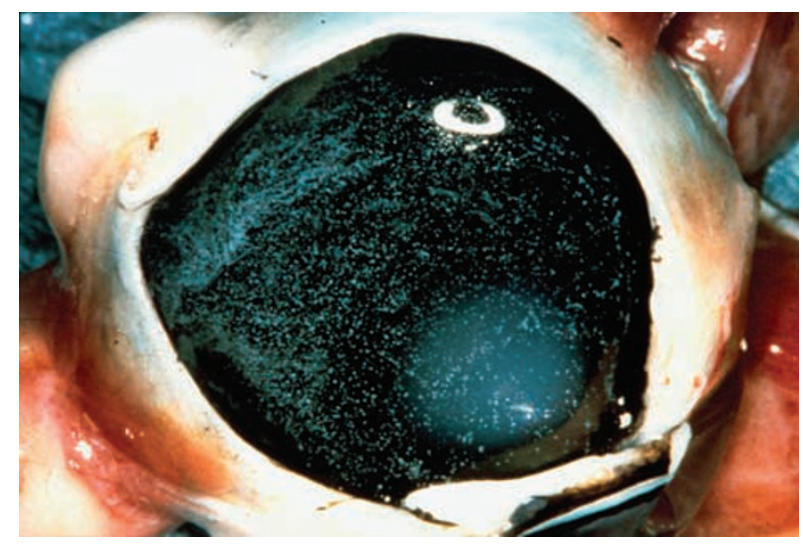

Figure 1 Appearance of $\mathrm{AH}$ in the dog vitreous. cataract, retinopathy, and keratopathy. The advantage of galactose feeding is that it induces aldose-reductase associated lesions in a non-diabetic animal within an earlier time frame than in the diabetic animal. ${ }^{36}$ Galactose-fed dogs that develop retinal vascular changes identical to diabetic dogs are more healthy, vigorous, and capable of a longer lifespan than their diabetic counterparts whose fragile lives must be maintained by insulin injections. This has the advantage of producing diabetes-like complications in a shorter time frame while maintaining the health of the animal. The galactose-fed $\operatorname{dog}$ is unique because it is the only animal model that demonstrates both the histological and clinical lesions associated with all stages of diabetic retinopathy, ranging from background to the proliferative stages. ${ }^{37-41}$ It is also the only animal model that develops bilateral $\mathrm{AH}$.

The time frame for diabetes-like ocular changes in initially 9-month-old galactose-fed beagles are summarized in Table 1 . These begin with the development of cataract within weeks after the initiation of the galactose diet. ${ }^{42-44}$ The development of retinal lesions follows with the degeneration of retinal capillary pericytes between 18 and 24 months, the histological development of microaneurysms (27-33 months), and the clinical appearance of dot and blot haemorrhages at 33-36 months. ${ }^{37,38,45}$ At this time, morphological changes in corneal endothelial cells also occur. ${ }^{46}$ Between 36 and 48 months, significant changes in retinal perfusion develop, which result in retinal hypoxia and the subsequent development of lesion association with the preproliferative and subsequently proliferative stage. ${ }^{38-41}$ It is during this time frame that $\mathrm{AH}$ appears.
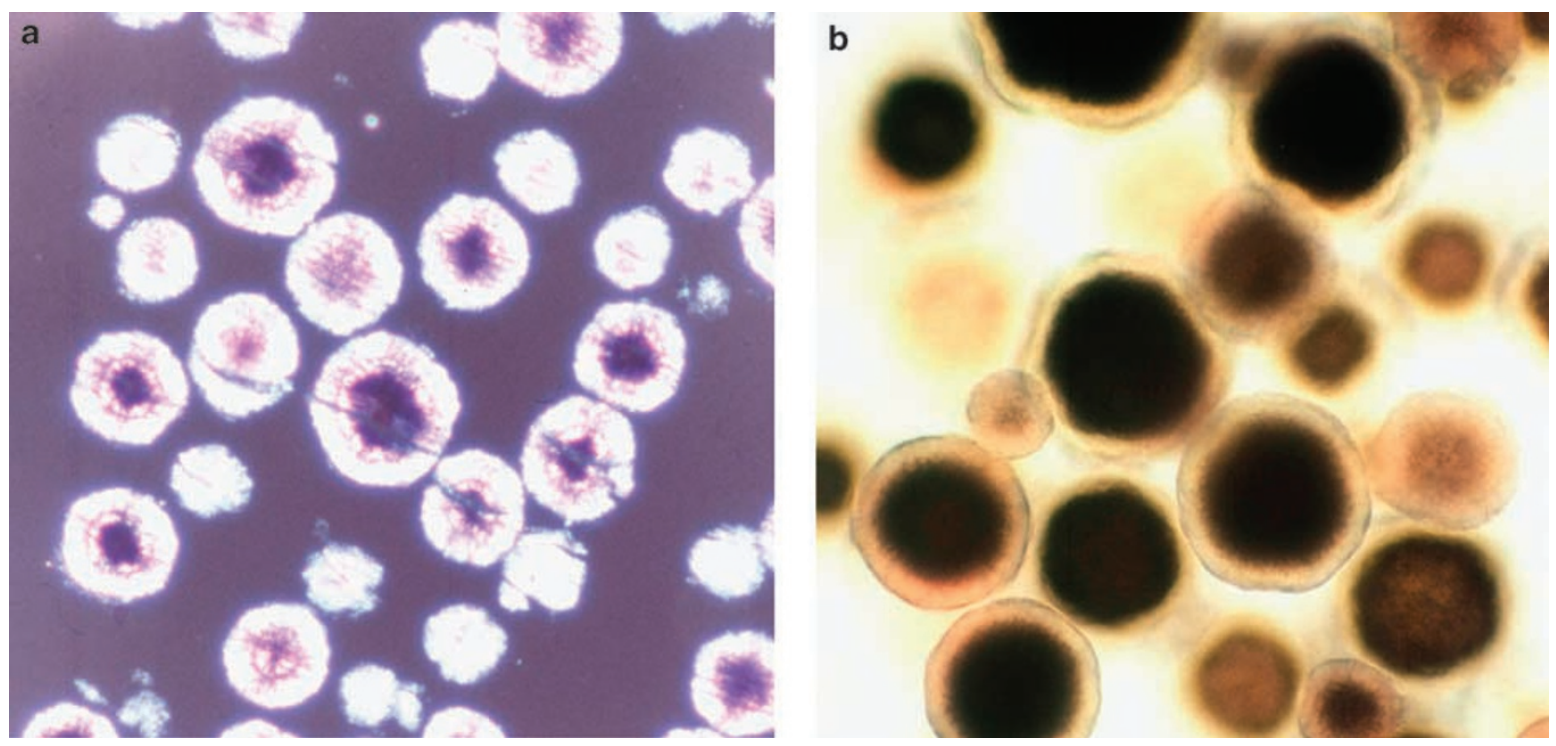

Figure 2 ABs from dog demonstrating birefringence (a) and lipid staining with oil red $\mathrm{O}(\mathrm{b})$. 
Figure 1 illustrates the appearance of $\mathrm{AH}$ in the vitreous of an enucleated eye from a galactose-fed beagle. Attempts at physically extracting the white ABs suspended in the viscous vitreous humour without contamination from the adhering vitreous were unsuccessful. Therefore, vitreous 'smears' containing ABs were prepared and examined by light microscopy. The ABs demonstrated birefringence (Figure 2a) and stained positive for lipids (Oil Red O) (Figure 2b). Subsequently, samples of the vitreous from dogs with and without $\mathrm{AH}$ were lyophilized and subjected to elemental analysis that indicated that the percent of calcium and phosphorus were significantly higher in the vitreous containing $A B s$ (Figure 3 ), thus verifying that dog ABs also contain calcium and phosphorus. A key feature of the eyes of galactose-fed dogs is the prolonged

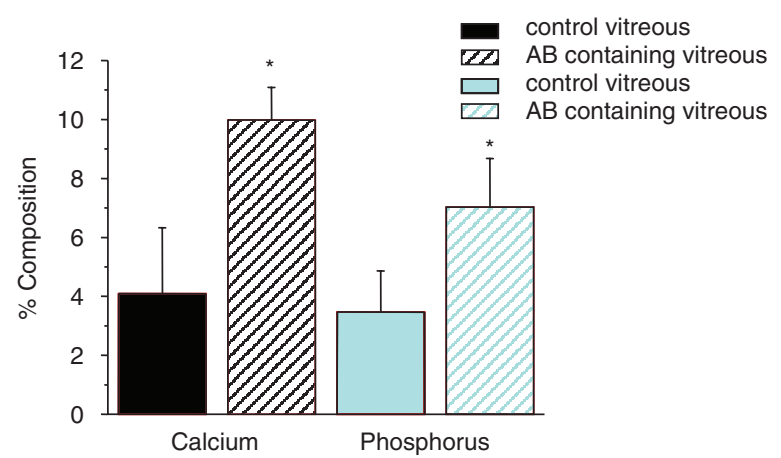

Figure 3 Results of elemental analysis of calcium and phosphorus of vitreous with/without AH from dogs. $n=3-4$, mean $\pm \mathrm{SD},\left({ }^{*} P<0.05\right)$.

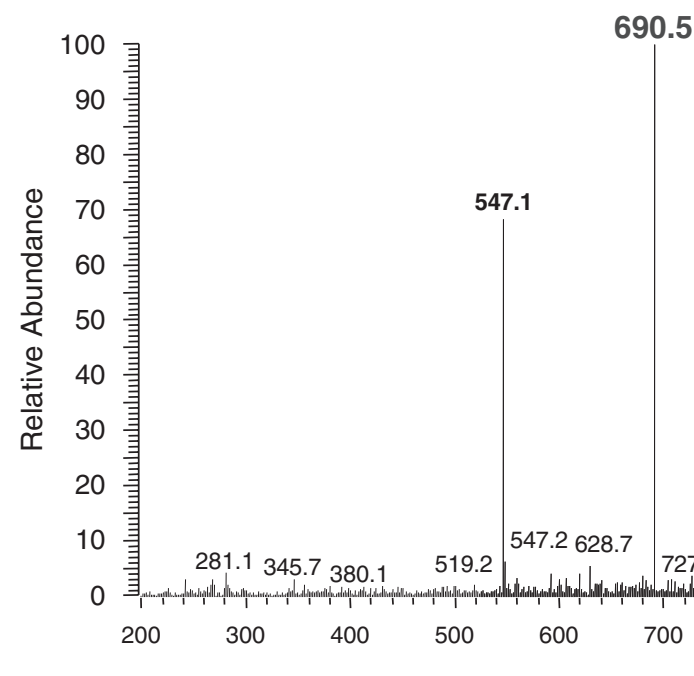

Figure 4 Subtraction spectrum of electrospray (ESI-MS) conducted in the negative mode on samples of vitreous with/without AH. Samples were directly infused into the heated capillary of the detector at $1.5 \mu \mathrm{l} / \mathrm{min}$. Spray voltage was $5 \mathrm{kV}$, temperature of the capillary was $275^{\circ} \mathrm{C}$; capillary voltage and tube lens offset voltage were both set up at $-30 \mathrm{~V}$. The mass range was 150-2000. Results show the presence of three peaks that are absent in vitreous without $\mathrm{AH}$. presence of mature cortical cataracts. Since mature sugar cataracts are more permeable and lens resorption is known to occur in dogs, ${ }^{47-49}$ the possibility that ABs contain lens crystallins was investigated. Vitreous with/ without $\mathrm{AH}$ was subjected to polyacrylamide gel electrophoresis and subjected to western blot analysis using antibodies against bovine $\alpha-, \beta-$, and $\gamma$-crystallins. This technique failed to show the presence of crystallins in the vitreous containing $\mathrm{AH}$.

To identify potential components present in the ABs, electrospray mass spectroscopy (ESI-MS) studies were conducted using a Finnigan $\mathrm{LCQ}^{\mathrm{TM}}$ mass spectrometer (ThermoQuest; San Jose, CA, USA). Since the ABs are impossible to separate from the vitreous humour, identical studies were conducted with the normal and the vitreous-containing $\mathrm{AH}$. The vitreous samples were sonicated in HPLC grade water using a Sonicator ${ }^{\mathbb{B}}$ ultrasonic processor (Misonix Inc., Farmingdale, NY, USA) for 1-2 $\mathrm{min}$, and the homogenate was then extracted three times with HPLC grade chloroform. Following removal of chloroform in vacuo, the residue was dissolved in HPLC grade methanol for mass spectroscopic analysis. Peaks in the extract associated with $\mathrm{ABs}$ were identified by subtraction analysis of vitreous with/without $\mathrm{AH}$. These analyses indicated the presence of three distinct peaks at $m / z$ peak 690.5, 547.1, and 1430.6, respectively (Figure 4). The major $m / z 690.5$ peak was identified by computer analysis as the quasimolecular ion of 1,2-dipalmitoyl-glycero-3using a relative collision energy of $34 \mathrm{eV}$ and comparing these results to a similar MS/MS analysis of standard phosphoethanolamine (DPPE) and confirmed by MS/MS

The $\mathrm{m} / \mathrm{z} 690.5$ ion is the quasimolecular ion of 1,2-dipalmitoyl-glycero-3-phosphoethanolamine (DPPE)

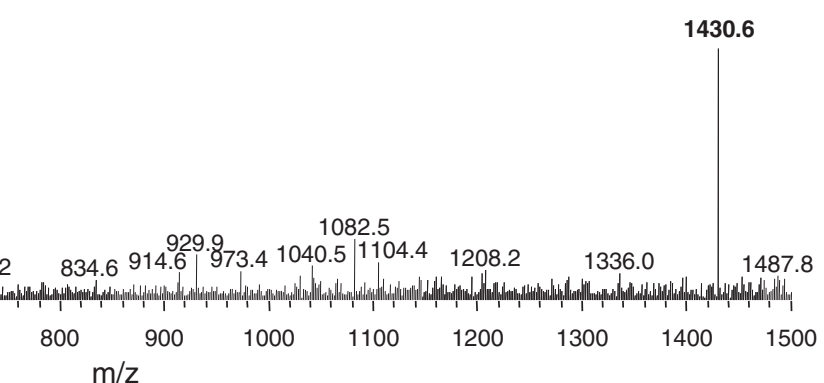




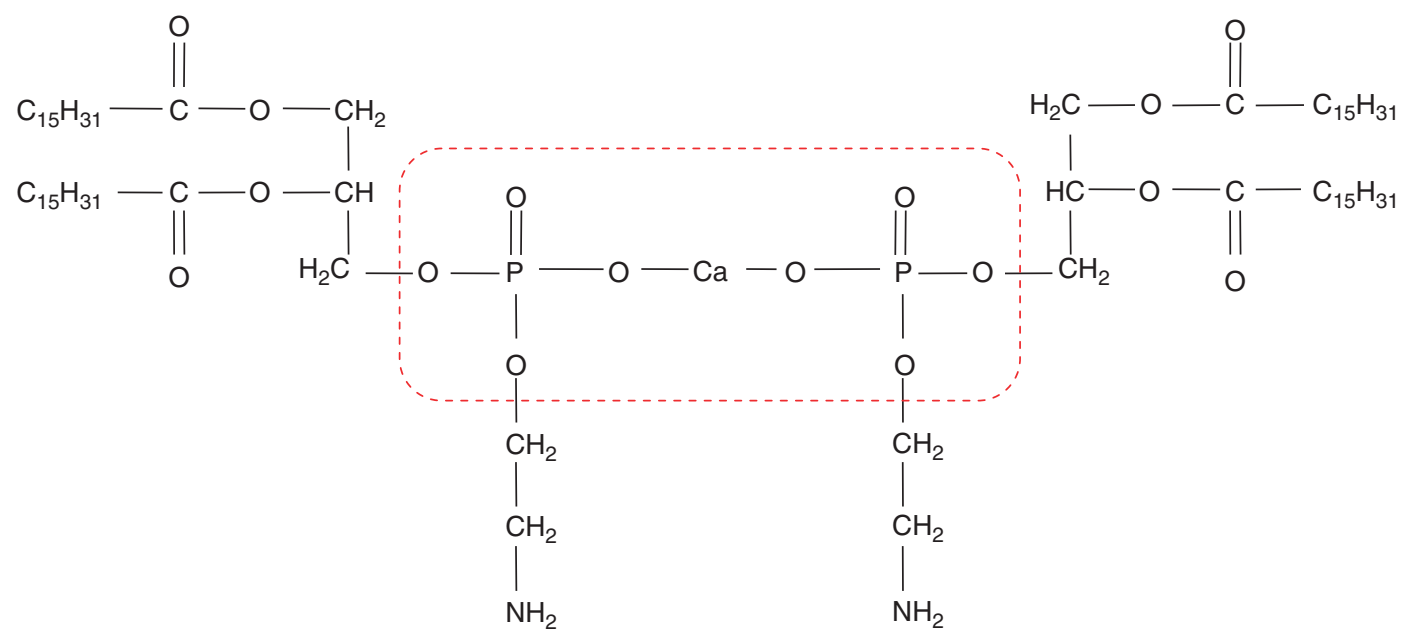

Figure 5 Structure of hydroxyapatite complex of 1,2-DPPE identified in ABs.

DPPE (Sigma-Aldrich). The identity of the $m / z 547.1$ and 1430.6 peaks remain unknown. On the basis of the identification of DPPE, the presence of Ca and P in ABs, and the suggested structural and elemental similarity of the $\mathrm{AB}$ with hydroxyapatite, the major component of the $\mathrm{AH}$ body is proposed to be a complex between two molecules of DPPE and calcium as outlined in Figure $5 .^{35}$

Since there are no reports on the presence of DPPE in the eye, where can the components of this phospholipid come from? Palmitic acid is the most abundant free fatty acid present in bovine and rabbit aqueous humour, and in the lens its metabolism to $\mathrm{CO}_{2}$ and glutamic acid generates energy. ${ }^{50}$ In the retina, palmitate along with other free fatty acids are known to increase with diabetes, and increased levels of retinal palmitate can initiate apoptosis of retinal capillary pericytes. ${ }^{51}$ Palmitic acid is also a major saturated free fatty acid present in the vitreous of humans and $\operatorname{dogs},{ }^{52}$ and its concentration has been shown to increase with proliferative retinopathy. ${ }^{53}$ Phosphatidylethanolamine has been reported to be the only phospholipid that is present at higher concentrations in the vitreous than in serum. ${ }^{54}$ This increase has been suggested to be due to direct diffusion from the retina. ${ }^{54}$ The retina contains relatively high amounts of glycerophosphorylethanolamine, ${ }^{55}$ and both diabetes and galactosemia are associated with both increased palmitic acid incorporation into diacylglycerol and increased levels of diacylglycerol. ${ }^{56}$ The elevated levels of diacylglycerol, in turn, can increase PKC activity. ${ }^{57}$

Since $\mathrm{AH}$ in the galactose-fed dog appears during a period in which significant retinal perfusion changes occur, we propose that the DPPE observed in the ABs of the galactosemic dogs may be derived from the retina and linked to the formation of retinal changes in these dogs. Diffusion of substances from the degenerating retina into the vitreous have been established. For example, evidence suggests that in the Royal College of Surgeons (RCS), posterior subcapsular cataracts (PSC) are initiated by toxic products generated by the peroxidation of polyunsaturated lipid components from the degenerating photoreceptor outer segments in the retina that diffuse anterior through the vitreous to the lens. ${ }^{58}$ Active lipid metabolism has also been reported to occur in the vitreous from Irish setters with hereditary rod-cone dysplasia (RCD). ${ }^{52} \mathrm{AH}$ has also been documented to be present in patients with retinitis pigmentosa, ${ }^{59-61}$ or pigmentary retinal degeneration. ${ }^{62}$

In addition to retinal changes, other factors appear to influence the development of $\mathrm{AH}$ in dogs. In studies in which dogs were made aphakic by phacoemulsification surgery before galactose feeding, ${ }^{63} \mathrm{AH}$ was clinically observed to occur after 25 months of galactose feeding. Moreover, it appeared similarly in dogs treated with aldose-reductase inhibitor, which was observed to significantly delay the development of retinal lesions and reduce the areas of retinal nonperfusion. Since surgery results in an inflammatory response, the ABs in these animals may be different. Unfortunately, the vitreous from these animals was not available for analysis.

These results clearly indicate that $\mathrm{AH}$ is a complex lesion associated with a number of biochemical changes in the eye. Although currently there are no prospects for its prevention, investigations on the development of $\mathrm{AH}$ in the galactose-fed dog animal model should provide an insight into the pathogenesis, future management and treatment of this ocular lesion. 


\section{References}

1 Bishop PN. Structural macromolecules and supramolecular organisation of the vitreous gel. Prog Retin Eye Res 2000; 19(3): 323-344.

2 Ueno N. [Changes in vitreous structure caused by oxygen free radicals]. Nippon Ganka Gakkai Zasshi 1995; 99(12): 1342-1360.

3 Takahashi K, Arai K, Hayashi S, Tanaka Y. Degree of degraded proteoglycan in human vitreous and the influence of peroxidation]. Nippon Ganka Gakkai Zasshi 2006; 110(3): 171-179.

4 Fawzi AA, Vo B, Kriwanek R, Ramkumar HL, Cha C, Carts A et al. Asteroid hyalosis in an autopsy population: The University of California at Los Angeles (UCLA) experience. Arch Ophthalmol 2005; 123(4): 486-490.

5 Mitchell P, Wang MY, Wang JJ. Asteroid hyalosis in an older population: the Blue Mountains Eye Study. Ophthalmic Epidemiol 2003; 10(5): 331-335.

6 Moss SE, Klein R, Klein BE. Asteroid hyalosis in a population: the Beaver Dam Eye Study. Am J Ophthalmol 2001; 132(1): 70-75.

7 Smith JL. Asteroid hyalitis: incidence of diabetes mellitus and hypercholesteremia. J Am Med Assoc 1958; 168(7): 891-893.

8 Wasano T, Hirokawa H, Tagawa H, Trempe CL, Buzney SM. Asteroid hyalosis: posterior vitreous detachment and diabetic retinopathy. Ann Ophthalmol 1987; 19(7): 255-258.

9 Luxenberg M, Sime D. Relationship of asteroid hyalosis to diabetes mellitus and plasma lipid levels. Am J Ophthalmol 1969; 67(3): 406-413.

10 Jones WL, Twamley CR. Documented acquired asteroid hyalosis in a case of early diagnosed diabetes mellitus. Optometry 2001; 72(5): 315-321.

11 Akram A, Niazi MK, Ishaq M, Azad N. Frequency of diabetics in asteroid hyalosis patients. J Ayub Med Coll Abbottabad 2003; 15(3): 10-11.

12 Topilow HW, Kenyon KR, Takahashi M, Freeman HM, Tolentino FI, Hanninen LA. Asteroid hyalosis. Biomicroscopy, ultrastructure, and composition. Arch Ophthalmol 1982; 100(6): 964-968.

13 Stanley JA, Martin DG. Asteroid hyalosis and the blood glucose. N C Med J 1970; 31(5): 183-185.

14 Bergren RL, Brown GC, Duker JS. Prevalence and association of asteroid hyalosis with systemic diseases. Am J Ophthalmol 1991; 111(3): 289-293.

15 Bard LA. Asteroid hyalitis: relationship to diabetes and hypercholesterolemia. Am J Ophthalmol 1964; 58: 239-242.

16 Hatfield RE, Gastineau CF, Rucker CW. Asteroid bodies in the vitreous: relationship to diabetes and hypercholesterolemia. Proc Staff Meet Mayo Clin 1962; 37: 513-514.

17 Yazar Z, Hanioglu S, Karakoc G, Gursel E. Asteroid hyalosis. Eur J Ophthalmol 2001; 11(1): 57-61.

18 Jervey ED, Anderson Jr WB. Asteroid hyalitis: a study of serum calcium levels in affected patients. South Med J 1965; 58: 191-194.

19 Lamba PA, Shukla KN. Vascular factors in asteroid hyalopathy. Clinical and biochemical observations. Can J Ophthalmol 1971; 6(1): 46-51.

20 Dogru M, Inoue M, Nakamura M, Yamamoto M. Modifying factors related to asymmetric diabetic retinopathy. Eye 1998 12(Part 6): 929-933.
21 Verhoeff FH. Microscopic findings in a case of asteroid hyalitis. Am J Opthalmol 1921; 4: 155-160.

22 Rodman HI, Johnson FB, Zimmerman LE. New histopathological and histochemical observations concerning asteroid hyalitis. Arch Ophthalmol 1961; 66: 552-563.

23 March WF, Shoch D. Electron diffraction study of asteroid bodies. Invest Ophthalmol 1975; 14(5): 399-400.

24 Streeten BW. Vitreous asteroid bodies. Ultrastructural characteristics and composition. Arch Ophthalmol 1982; 100(6): 969-975.

25 Adenis JP, Leboutet MJ, Loubet R. [Asteroid hyalopathy. Ultrastructural study of 3 cases]. J Fr Ophtalmol 1984; 7(8-9): 529-534.

26 Miller H, Miller B, Rabinowitz H, Zonis S, Nir I. Asteroid bodies - an ultrastructural study. Invest Ophthalmol Vis Sci 1983; 24(1): 133-136.

27 Winkler J, Lunsdorf $H$. Ultrastructure and composition of asteroid bodies. Invest Ophthalmol Vis Sci 2001; 42(5): 902-907.

28 Komatsu H, Kamura Y, Ishi K, Kashima Y. Fine structure and morphogenesis of asteroid hyalosis. Med Electron Microsc 2003; 36(2): 112-119.

29 Feldman GL. Human ocular lipids: their analysis and distribution. Surv Ophthalmol 1967; 12(3): 207-243.

30 Lin SY, Chen KH, Cheng WT, Ho CT, Wang SL. Preliminary identification of beta-carotene in the vitreous asteroid bodies by micro-Raman spectroscopy and HPLC analysis. Microsc Microanal 2007; 13(2): 128-132.

31 Schaffer EH. [Asteroid hyalosis in the dog]. Tierarztl Prax 1985; 13(1): 71-75.

32 Fritsche J. Asteroide Hyalose bei Hund und Katze-eine ophthalmopathologische Untersuchung Diss. München University, 1995; http:/ /library.vetmed.fu-berlin.de/ ResourceList/details/64868.

33 Peiffer RL, Johnson PT. Clinical ocular findings in a colony of chinchillas (Chinchilla laniger). Lab Anim 1980; 14(4): 331-335.

34 Zauberman H, Livni N. Experimental vascular occlusion in hypercholesterolemic rabbits. Invest Ophthalmol Vis Sci 1981; 21(2): 248-255.

35 Wang M, Kador PF, Wyman M. Structure of asteroid bodies in the vitreous of galactose-fed dogs. Mol Vis 2006; 12: 283-289.

36 Kinoshita JH, Kador P, Catiles M. Aldose reductase in diabetic cataracts. JAMA 1981; 246(3): 257-261.

37 Kador PF, Akagi Y, Takahashi Y, Ikebe H, Wyman M, Kinoshita JH. Prevention of retinal vessel changes associated with diabetic retinopathy in galactose-fed dogs by aldose reductase inhibitors. Arch Ophthalmol 1990; 108(9): 1301-1309.

38 Kador PF, Takahashi Y, Akagi Y, Neuenschwander H, Greentree W, Lackner $\mathrm{P}$ et al. Effect of galactose diet removal on the progression of retinal vessel changes in galactose-fed dogs. Invest Ophthalmol Vis Sci 2002; 43(6): 1916-1921.

39 Kador PF, Takahashi Y, Wyman M, Ferris III F. Diabetes-like proliferative retinal changes in galactose-fed dogs. Arch Ophthalmol 1995; 113(3): 352-354.

40 Takahashi Y, Wyman M, Ferris III F, Kador PF. Diabetes-like preproliferative retinal changes in galactose-fed dogs. Arch Ophthalmol 1992; 110(9): 1295-1302.

41 Neuenschwander H, Takahashi Y, Kador PF. Dosedependent reduction of retinal vessel changes associated with diabetic retinopathy in galactose-fed dogs by the 
aldose reductase inhibitor M79175. J Ocul Pharmacol Ther 1997; 13(6): 517-528.

42 Kador PF, Betts D, Wyman M, Blessing K, Randazzo J. Effects of topical administration of an aldose reductase inhibitor on cataract formation in dogs fed a diet high in galactose. Am J Vet Res 2006; 67(10): 1783-1787.

43 Sato S, Takahashi Y, Wyman M, Kador PF. Progression of sugar cataract in the dog. Invest Ophthalmol Vis Sci 1991; 32(6): 1925-1931.

44 Sato S, Secchi EF, Lizak MJ, Fukase S, Ohta N, Murata M et al. Polyol formation and NADPH-dependent reductases in dog retinal capillary pericytes and endothelial cells. Invest Ophthalmol Vis Sci 1999; 40(3): 697-704.

45 Takahashi Y, Augustin W, Wyman M, Kador PF. Quantitative analysis of retinal vessel changes in galactosefed dogs. J Ocul Pharmacol 1993; 9(3): 257-269.

46 Datiles MB, Kador PF, Kashima K, Kinoshita JH, Sinha A. The effects of sorbinil, an aldose reductase inhibitor, on the corneal endothelium in galactosemic dogs. Invest Ophthalmol Vis Sci 1990; 31(11): 2201-2204.

47 Lackner PA, Rodriguez L, Sato S, Lizak MJ, Wyman M, Kador PF. Age-dependent lens changes in galactose-fed dogs. Exp Eye Res 1997; 64(3): 431-436.

48 Basher AW, Roberts SM. Ocular manifestations of diabetes mellitus: diabetic cataracts in dogs. Vet Clin North Am Small Anim Pract 1995; 25(3): 661-676.

49 Gonzalez-Alonso-Alegre E, Rodriguez-Alvaro A. Spontaneous resorption of a diabetic cataract in a geriatric dog. I Small Anim Pract 2005; 46(8): 406-408.

50 van Heyningen R, Linklater J. Metabolism of $14 \mathrm{C}$ palmitic acid by the lens. Invest Ophthalmol 1976; 15(5): 427-430.

51 Cacicedo JM, Benjachareowong S, Chou E, Ruderman NB, Ido Y. Palmitate-induced apoptosis in cultured bovine retinal pericytes: roles of $\mathrm{NAD}(\mathrm{P}) \mathrm{H}$ oxidase, oxidant stress, and ceramide. Diabetes 2005; 54(6): 1838-1845.

52 Reddy TS, Birkle DL, Packer AJ, Dobard P, Bazan NG. Fatty acid composition and arachidonic acid metabolism in vitreous lipids from canine and human eyes. Curr Eye Res 1986; 5(6): 441-447.

53 Kishikawa Y, Gong H, Kitaoka T, Amemiya T, Takaya K, Tozu $\mathrm{M}$ et al. Elements and organic substances in epiretinal proliferative tissue excised during vitreous surgery: analysis by time-of-flight secondary-ion mass spectrometry.

J Electron Microsc (Tokyo) 2003; 52(3): 349-354.

54 Kim JO, Cotlier E. Phospholipid distributions and fatty acid composition of lysophosphatidylcholine and phosphatidylcholine in rabbit aqueous humor, lens and vitreous. Exp Eye Res 1976; 22(6): 569-576.

55 Alberghina M, Giacchetto A, Cavallaro N. Levels of ethanolamine intermediates in the human and rat visual system structures: comparison with neural tissues of a lower vertebrate (Mustelus canis) and an invertebrate (Loligo pealei). Neurochem Int 1993; 22(1): 45-51.

56 Xia P, Inoguchi T, Kern TS, Engerman RL, Oates PJ, King GL. Characterization of the mechanism for the chronic activation of diacylglycerol-protein kinase C pathway in diabetes and hypergalactosemia. Diabetes 1994; 43(9): 1122-1129.

57 Goldberg EM, Zidovetzki R. Effects of dipalmitoylglycerol and fatty acids on membrane structure and protein kinase $\mathrm{C}$ activity. Biophys J 1997; 73(5): 2603-2614.

58 Zigler Jr JS, Hess HH. Cataracts in the Royal College of Surgeons rat: evidence for initiation by lipid peroxidation products. Exp Eye Res 1985; 41(1): 67-76.

59 Wafi M, Rais L, Lahbil D, Hamdani M, Rachid R, Belhadji M et al. [A case of a retinitis pigmentosa and asteroid hyalosis]. J Fr Ophthalmol 2004; 27(7): 801-804.

60 van den Born LI, van Soest S, van Schooneveld MJ, Riemslag FC, de Jong PT, Bleeker-Wagemakers EM. Autosomal recessive retinitis pigmentosa with preserved para-arteriolar retinal pigment epithelium. Am J Ophthalmol 1994; 118(4): 430-439.

61 Yin C. A case of retinitis pigmentosa and asteroid hyalosis: diagnosis and low vision management. J Am Optom Assoc 1984; 55(10): 773-776.

62 Sebag J, Albert DM, Craft JL. The Alstrom syndrome: ophthalmic histopathology and retinal ultrastructure. $\mathrm{Br} J$ Ophthalmol 1984; 68(7): 494-501.

63 Cusick M, Chew EY, Ferris III F, Cox TA, Chan CC, Kador PF. Effects of aldose reductase inhibitors and galactose withdrawal on fluorescein angiographic lesions in galactosefed dogs. Arch Ophthalmol 2003; 121(12): 1745-1751. 